\title{
Irrational Beliefs in Employees with an Adjustment, a Depressive, or an Anxiety Disorder: a Prospective Cohort Study
}

\author{
Karen Nieuwenhuijsen · Jos H. A. M. Verbeek • \\ Angela G. E. M. de Boer · Roland W. B. Blonk • \\ Frank J. H. van Dijk
}

Published online: 27 February 2008

(C) The Author(s) 2007

\begin{abstract}
It remains unclear if patients with different types of common mental disorders, such as adjustment, anxiety and depressive disorders, have the same irrational ideas. The aim of this prospective cohort study $(n=190)$ is to investigate differences in level and type of irrational beliefs among these groups and to examine whether a change in irrational beliefs is related to symptom recovery. Irrational beliefs (IBI) and symptoms were measured at four points in time: at baseline, after 3, 6 and 12 months. Results showed that diagnostic groups differed in their level of irrational beliefs and this effect remained over time. Highest levels of irrationality were observed in the double diagnosis group, followed by the anxiety disorder group and the depression group. Participants with adjustment disorders showed the lowest levels of irrationality, comparable to a community sample. We did not find differences in the type of irrational beliefs between diagnostic groups. The level of irrationality declined over time for all diagnostic groups. No differences in decrease were observed between diagnostic groups. The magnitude and direction of change in irrational beliefs were related to the magnitude of recovery of depressive, anxiety
\end{abstract}

K. Nieuwenhuijsen $(\bowtie)$ · J. H. A. M. Verbeek · A. G. E. M. de Boer · F. J. H. van Dijk Coronel Institute of Occupational Health, Academic Medical Center, University of Amsterdam, P.O. Box 22700, 1100 DD Amsterdam, The Netherlands

e-mail: k.nieuwenhuijsen@amc.nl

J. H. A. M. Verbeek

e-mail: j.h.verbeek@amc.nl

A. G. E. M. de Boer

e-mail: a.g.deboer@amc.nl

F. J. H. van Dijk

e-mail: f.j.vandijk@amc.nl

R. W. B. Blonk

TNO Quality of Life, Hoofddorp, The Netherlands

e-mail: Roland.Blonk@tno.nl 
and stress symptoms over time. These results support the application of general cognitive interventions, especially for patients with a depressive or an anxiety disorder.

Keywords Irrational beliefs - Cognitive therapy - Common mental disorders · Follow-up studies

\section{Introduction}

In recent years, cognitive behaviour therapy (CBT) has been applied to a broad variety of patient groups and settings. It is advocated as the therapy of choice for common mental disorders, such as anxiety and depressive disorders, by the Department of Health of the UK (Department of Health 2001). The cognitive component of CBT is based upon the notion that irrational cognitions play a key role in the development and maintenance of emotional disturbances (Beck 1976; Ellis 1977). According to this theory, emotions are not wholly determined by environmental events but rather, negative emotions are enhanced by irrational cognitions, which magnify the intensity of the situation at hand. For example, the prominent model of Rational Emotive Behaviour Therapy (REBT) in cognitive therapy postulates that excessive negative emotions are the result of irrational beliefs such as "I must absolutely not fail, I cannot tolerate failing". Within the framework of cognitive therapy, symptom recovery is achieved by disputing these irrational beliefs and replacing them with more adaptive rational cognitions (Ellis 1995). Reviews of the efficacy of REBT have shown that it is an effective treatment (e.g., Engels et al. 1993; Lyons and Woods 1991).

For clinical reasons, it would be relevant to know whether patients with different types of common mental disorders, such as adjustment disorders, anxiety and depression, are equally irrational. Irrationality may differ in many aspects. Patients with one disorder may endorse an overall higher level of irrational beliefs than patients with other disorders. Knowledge of the level of irrationality in different diagnoses could provide clinicians with clues as to which patients could potentially benefit most from cognitive interventions. Furthermore, patients with different diagnoses may endorse different types of irrational cognitions. Such information would generate indications of which irrational ideas should be addressed during CBT. Finally, irrational beliefs may be more persistent in certain disorders. Along with the level of irrationality, persistency of irrationality over time could be an indicator of the severity of the problem of irrationality in certain disorders. For clinicians, such knowledge could inform their choice of intensity of their treatment for certain diagnostic groups.

One might expect patients with less severe disorders to have lower level of irrational beliefs compared to patients with more severe disorders. Within the group of common mental disorders adjustment disorders are considered less severe than depressive and anxiety disorders in terms of the DSM-IV classification (APA 1994). However, we do not know of any empirical evidence of a difference in level of irrationality between adjustment disorders and depressive or anxiety disorders. In 
non-clinical populations, the level of irrationality has been studied in relation to different symptoms. Several studies have found that level of irrational beliefs are associated with depressive symptoms (Chang and Bridewell 1998; Chang 1997; Kassinove and Eckhardt 1994) and anxiety symptoms (Malouff 1992). Support for differential effects of level of irrationality and specific symptoms is scarce. Chang (1997) found that level of irrational beliefs was related to depressive symptoms, but not to life stress. Moreover, Chang and Bridewell (1998) found that a higher level of irrational beliefs was found in students with depressive symptoms compared to students with anxiety symptoms. In conclusion, there is a need for evidence of a relation between severity of the disorder and the level of irrational beliefs that comes from research in clinical populations.

Although the relationship between irrational beliefs and negative feelings is a core component of REBT, the exact impact of irrational beliefs on emotion has not yet been clarified (David et al. 2005). It remains unclear whether specific irrational beliefs lead to specific symptoms and specific disorders, as opposed to the possibility that any irrational belief is capable to trigger any symptom. For instance, emotional irresponsibility (attributing emotions to external causes rather than to oneself) can be hypothesised to trigger depressive symptoms especially. Another example might be that worrying is mainly linked to anxiety symptoms. Although common mental disorders may have symptoms in common, the core negative feelings associated with the disorders differ. Therefore, if the content of irrational beliefs is linked to the content of the symptom one would expect patients with different common mental disorders to have different types of irrational beliefs.

The question whether patients with different common mental disorders endorse different types of irrational beliefs has, to our knowledge, been the subject of one study only. A recent study by Taghavi and colleagues (2006) comparing depressed and anxious patients found some differences between types of irrational beliefs between depressed and anxiety patients. Using the Irrational Beliefs Test as a measure of irrationality, they found no difference between the two groups in 8 out of 10 of the subscales (demand approval [the need to be approved of and loved by all the significant people in one's life], high self-expectation [believing one must be thoroughly competent and adequate in all respects in order to be worthwhile], blame proneness [people who behave badly are awful and should be punished severely], frustrated reactivity [the idea that it is horrible if things are not as one would like], anxious overconcern, problem avoidance, dependency and helplessness for change [the idea that one is a helpless victim of the past]). However, emotional irresponsibility and perfectionism were significantly higher in the depressed group.

The relation between specific symptoms and the type of irrational beliefs has also been the subject of study in student populations (Chang and D'Zurilla 1996; Ciarrriochi 2004) as well as a community sample (Koopmans et al. 1994). The study of Ciarriochi (2004) examined the differential relations of types of irrational cognitions according to the Common Beliefs Survey-III with psychological symptoms in a student population. This study revealed that stress symptoms were best predicted by self-downing (the belief that one is worthless when not meeting their own standards or making a mistake) and need for approval, anxiety symptoms were best predicted by demanding perfection and need for approval, and finally 
depression was best predicted by self-downing and demanding perfection. Chang and D'Zurilla (1996) used the Survey of Personal Beliefs and found that low frustration tolerance was related to both anxiety and depressive symptoms. Otherdirected shoulds (inflexible demands of others) and self-worth however (negative self-rating) were only related to anxiety symptoms. Awfulizing (overestimating the seriousness of an event) and self-directed shoulds (inflexible self-demands) were not related to either symptom. The study by Koopmans and colleagues (1994) found higher correlations between worrying and need for approval measured by the Irrational Beliefs Inventory and anxiety compared to the correlations of these subscales with depressive symptoms. Correlations with the subscales emotional irresponsibility and problem avoidance were similar for both types of symptoms. Rigidity was not correlated to either depressive or anxiety symptoms.

The picture that arises from these clinical and non-clinical studies is not clear due to use of different populations, different instruments to measure irrational beliefs and different methods for the statistical analysis. The two irrational beliefs that are linked to a specific symptom or disorder in more than one study are: need for approval (anxiety) and emotional irresponsibility (depression). We argue that more studies in clinical populations are needed to explore the presence of specific irrational beliefs in different common mental disorders and to confirm the specificity of need for approval and emotional irresponsibility in anxiety and depression, respectively.

We examined irrational beliefs as part of a longitudinal cohort study of workers with common mental disorders. This enabled us to compare not only level and type of irrational beliefs between diagnostic groups, but also the stability of these features over time. Therefore, the first aim of this study is to investigate differences in level and type of irrational beliefs among a population of patients with adjustment, depressive, or anxiety disorders. Differences in level and type will be investigated using analysis of variance of total IBI-scores (level) and IBI-subscales (type) at baseline and over time with diagnosis as between-subjects factor.

More knowledge on the association between changes in irrational beliefs and changes in symptoms could provide important evidence for the basic premises of REBT theory (David et al. 2005). Few studies have directly tested the REBT theory of change. Intervention studies evaluating the efficacy of REBT have found that REBT leads to both symptom recovery and less irrational beliefs in patients with social anxiety (Emmelkamp et al. 1985), obsessive-compulsive disorder (Emmelkamp and Beens 1991) and a mixed population of neurotic disorders (Lipsky et al. 1980). However, these studies had fairly small sample sizes (ranging from 31 to 50 patients). Moreover, these studies did not test whether the decrease in irrational beliefs was related to the (magnitude of) decrease in symptoms. Such information could further underpin the REBT theory of change. A review by Oei and Free (1995) did find that in their review of various treatments for depression that the degree of change in irrational beliefs was related to the change in depression. Although our cohort was not specifically treated with REBT, but its longitudinal design and heterogeneous sample does provide an opportunity to examine the association between changes in irrational beliefs and changes in various symptoms. The second aim of this study is therefore to examine whether a change in irrational 
beliefs over time is related to symptom recovery in a cohort of patients with common mental disorders. We tested the hypothesis that the magnitude of the change in irrational beliefs is related to the magnitude of symptom change by comparing the symptom change in groups with different levels of change in irrational beliefs over time.

\section{Methods}

Participants and Procedure

As part of a longitudinal cohort study of employees with mental health problems, 30 occupational physicians from nine occupational health services provided data on consecutive patients. In order to include only new cases, eligible employees had to have been on full sick leave for less than 6 weeks due to mental health problems. Mental health problems were defined as psychological symptoms which were not caused by a somatic disorder. Any previous consultation with the occupational physician had to be more than 3 months previously. All participants underwent occupational rehabilitation provided by the occupational physician. According to their clinical guidelines, these physicians are expected to assess psychological symptoms and impairments of occupational functioning, evaluate whether treatment in the curative sector is needed and/or effective, assess impediments in the returnto-work process and carry out interventions aimed at the workplace or the individual. An example of an intervention aimed at the workplace is providing advice on a gradual return to work. The interventions aimed at the individual could include making an inventory of problems and stressors and consequently of solutions.

From March 2001 until February 2002, data on 277 employees eligible for participation in this study were reported to us by the occupational physicians. Of these 277 patients, 66 (24\%) refused to participate. Of the remaining 211 employees who signed an informed consent form, 198 filled out the baseline questionnaire. For the purpose of this study, only data from participants with common mental disorders (adjustment disorder, depression, anxiety disorder, or double diagnosis of depression and anxiety disorder) were used $(n=188)$.

Each participant was interviewed by the researchers by telephone. Subsequently, four questionnaires were sent to the participants by mail at baseline (t0), three (t 1$)$, six (t2) and 12 months (t3). One reminder was sent to each participant who did not return the questionnaire within 2 weeks.

\section{Measures}

\section{Diagnostic Interview}

Participants were diagnosed by means of a telephone version of the structured Composite International Diagnostic Interview (CIDI) (World Health Organization 
1997). An interview by telephone was used because of its convenience and its demonstrated comparability with face-to-face interviews (Simon et al. 1993; Rohde et al. 1997; Fenig et al. 1993). The telephone interview included the following diagnostic groups: major depressive disorder, panic disorder, social phobia, somatoform disorder, bipolar disorder, obsessive-compulsive disorder, posttraumatic stress disorder and psychotic disorder. For the first three diagnoses we administered the full CIDI scales, while we used only the stem (or screen) questions for the other categories. It was allowed for a participant to meet the criteria for more than one diagnosis. Anxiety disorder was operationalised as meeting the criteria for one or more of the following disorders: panic disorder, social phobia, somatoform disorder, obsessive-compulsive disorder, or post-traumatic stress disorder. Depression was operationalised as meeting the criteria for major depressive disorder. All interviews were conducted or supervised by a mental health professional. All interviews were tightly scripted and lasted 15-20 min.

\section{Questionnaire}

The baseline questionnaire provided information on age, gender, occupation (De Zwart et al. 1997) and self-reported cause of mental health problems, while irrational beliefs and symptoms were measured in all four questionnaires. The Irrational Beliefs Inventory (IBI) is a 50-item measure of irrational beliefs, comprising five subscales (Worrying, Rigidity, Need for Approval, Problem Avoidance and Emotional Irresponsibility) and a total score (Koopmans et al. 1994). Each item is scored on a 5-point Likert scale ranging from 1 (strongly disagree) to 5 (strongly agree). A high score reflects higher irrationality. Support for satisfactory internal consistency, construct validity and sensitivity for change was found (Koopmans et al. 1994; Emanuels-Zuurveen and Emmelkamp 1996). The total score was used to assess level of irrationality, while the five subscales provided information on the type of irrational beliefs that the participants endorsed.

Psychological symptoms were measured using the DASS-42, which consists of 42 items divided into three subscales: Depression scale, Anxiety scale and Stress scale (Lovibond and Lovibond 1993). Participants rated the extent to which they had experienced each symptom over the previous week on a 4-point Likert scale ranging from 0 (did not apply to me at all) to 3 (applied to me very much, or most of the time). Internal consistency (Brown et al. 1997; Clara et al. 2001), construct validity (Antony et al. 1998; Brown et al. 1997; Clara et al. 2001; Lovibond and Lovibond 1995; Nieuwenhuijsen et al. 2003), convergent and divergent validity (Antony et al. 1998; Brown et al. 1997; Lovibond and Lovibond 1995; Nieuwenhuijsen et al. 2003) and criterion validity (Nieuwenhuijsen et al. 2003) of the DASS were satisfactory. Missing values within the subscales of the DASS were imputed by the mean of the subscales if more than half of the items of that scale had been filled in. 


\section{Results}

At baseline, the internal consistency of the DASS subscales was high, with Cronbach's alpha's of .94, .88 and .93 for the Depression, Anxiety and Stress subscales, respectively. The internal consistencies of the IBI-subscale were somewhat less favourable with .81 for IBI-worrying, .81 for IBI-Rigidity, .86 for IBI-Need for Approval, .75 for IBI-problem avoidance and .77 for IBI-Emotional Irresponsibility.

\section{Employee Characteristics}

Table 1 presents the characteristics of the employees who participated in the study. Two of the original participants did not return any of the follow-up questionnaires (lost to follow-up). Overall, 53 (7\%) of the four questionnaires sent to each of the remaining 186 participants were missing. The maximum of missing data per subscale of the IBI and DASS was $18 \%$. A non-response analysis revealed no statistically significant differences in gender, IBI-total score, DASS-Depression, DASS-Anxiety and DASS-Stress scores at baseline between participants with and without missing questionnaires. Compared to participants without missing questionnaires, participants with one or more questionnaires missing were significantly younger (mean age 40 years vs. 45 years; $t=3.2, p<0.05$ ).

Gender differences in baseline IBI-scores were examined using one-way analysis of variance (ANOVA) on IBI-total scores and IBI-subscales at baseline. This analysis revealed that women showed statistically significant higher scores on IBIworrying (mean 39.6 compared to men who had a mean score of 37.3; $F(1,179)=5.8, p=0.02)$ but not on any of the other subscales nor IBI-total score.

Table 1 Mean, standard deviation (SD) or percentage of gender, age, diagnosis, occupation, and self-reported cause of common mental disorder. Due to missing values, $n$ ranges from 183 to 186

\begin{tabular}{lc}
\hline Participant characteristic & $\%(n)$ \\
\hline Gender, male & $40(74)$ \\
Age, mean (SD) & $44.5(8.5)$ \\
Diagnosis & \\
$\quad$ Adjustment disorder & $64(120)$ \\
$\quad$ Depression & $16(30)$ \\
$\quad$ Anxiety disorder & $12(22)$ \\
$\quad$ Both anxiety disorder and depression & $8(14)$ \\
$\quad$ (double diagnosis) & \\
Occupation & $44(80)$ \\
$\quad$ Mentally demanding (light and heavy) & $53(98)$ \\
$\quad$ Mixed mentally and physically demanding & $3(6)$ \\
$\quad$ Physically demanding (light and heavy) & $68(125)$ \\
Cause of common mental disorder & \\
$\quad$ (self-reported), work-related &
\end{tabular}




\section{Differences in Level of Irrationality between Diagnostic Groups}

To examine the overall difference in level of IBI total scores, one-way analysis of variance (ANOVA) was conducted on IBI scores at t0 between the four main diagnostic groups; adjustment disorder, depression, anxiety disorder and double diagnosis (both depression and anxiety disorder). This analysis revealed a main effect of diagnostic group for IBI-total scores: $(F(3,180)=10.3, p<0.001)$, indicating that the diagnostic groups had statistically different IBI-total scores at baseline. As can be seen in Table 2, employees with a double diagnosis had the highest mean IBI total scores, followed by employees with an anxiety disorder and employees with a depression. Patients with adjustment disorders had the lowest IBI-total scores.

Tukey post-hoc analysis indicated that the adjustment disorder group had statistically significant lower scores than either the anxiety disorder group ( $p=0.04)$ or the double diagnosis group $(p<0.001)$. Furthermore, the depression group scored significantly lower than the double diagnosis group $(p=0.001)$.

To examine the stability in level of IBI total scores over time, one-way analysis of variance with repeated measures (MANOVA) was conducted on IBI scores at t0 to $\mathrm{t} 3$ between the four main diagnostic groups; adjustment disorder, depression, anxiety disorder and double diagnosis (both depression and anxiety disorder). This analysis revealed a main effect of diagnostic group for IBI-total scores over time: $(F(3,441)=11.92, p<0.0001)$. This indicates that the diagnostic groups had statistically different IBI-total scores over time (Table 2). The mean scores of the IBI-total scores over time reveal that, as was found at baseline, patients with a double diagnosis had the highest IBI total scores, followed by employees with an anxiety disorder and employees with a depression. Employees with adjustment disorders had the lowest IBI-total scores. No statistically significant time $\times$ diagnosis interaction effect was found $(F(9,441)=0.62, p=0.78)$, indicating that the change in IBI-total scores over time was similar in all diagnostic groups (Fig. 1).

Tukey post-hoc analysis showed that the adjustment disorder group had statistically significant lower mean IBI total scores over time than either the anxiety disorder group ( $p=0.046)$ or the double diagnosis group $(p<0.0001)$. The difference between the adjustment disorder and the depression group was not statistically significant $(p=0.21)$. Furthermore, the difference between the depression group and the double diagnosis was statistically significant $(p=0.03)$. The differences in mean IBI-total scores over time between the anxiety disorder group on one hand and the depression group and the double diagnosis group on the other were both not statistically significant ( $p=0.90$ and $p=0.16$, respectively).

\section{Differences in Type of Irrational Beliefs between Diagnostic Groups}

To subsequently test the overall difference in type of irrational beliefs at t0 between the diagnostic groups, Multivariate Analysis of Variance (MANOVA) of the 


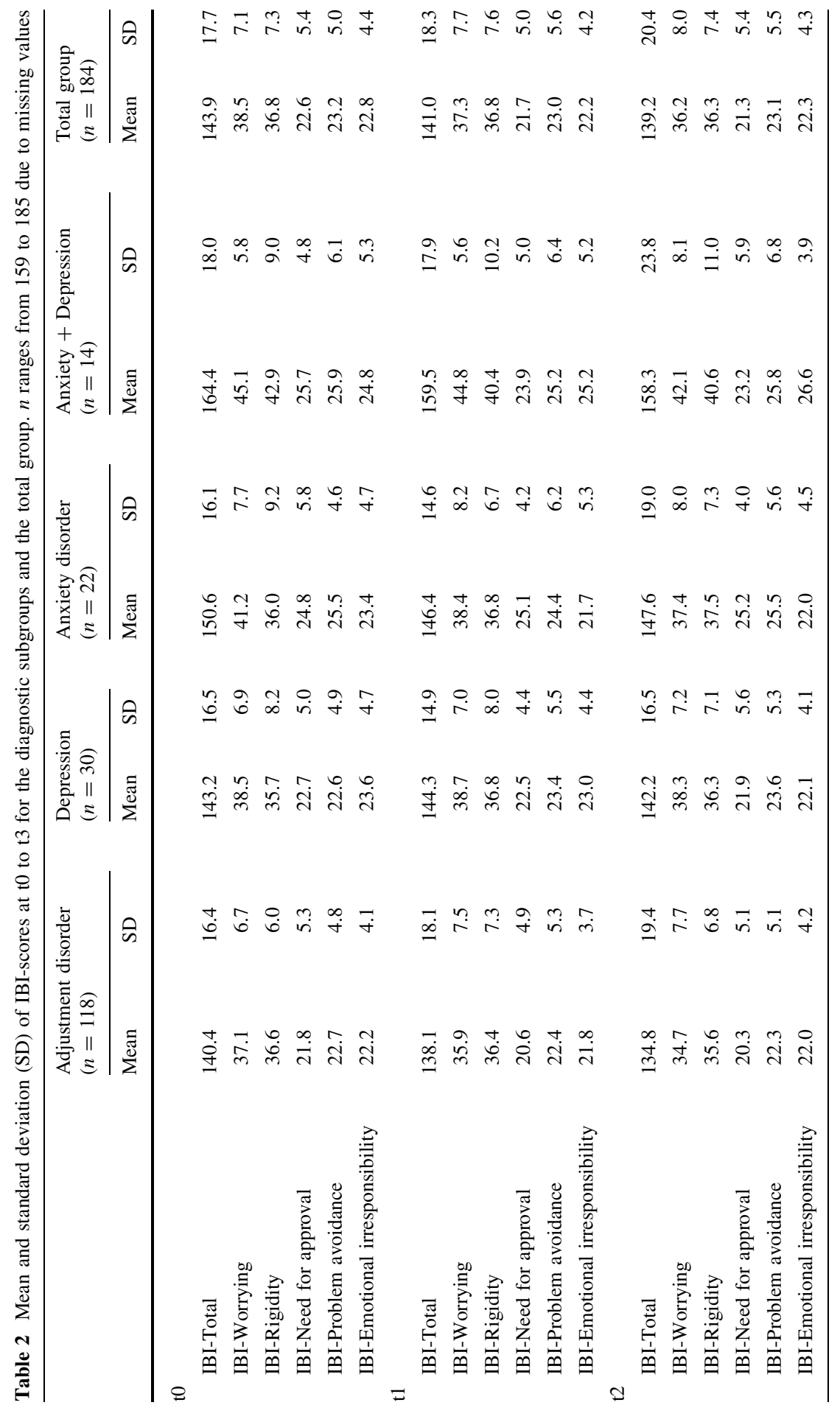









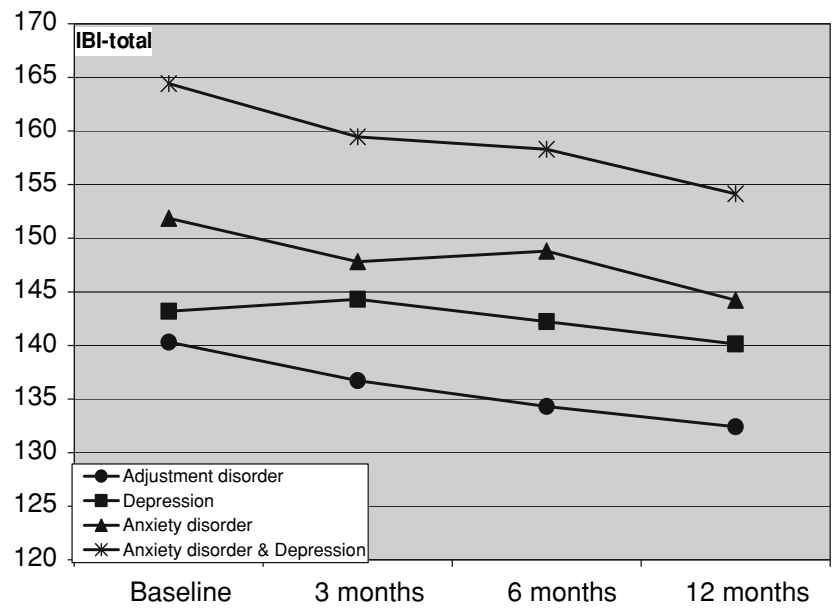

Fig. 1 Mean IBI-total scores at $\mathrm{t} 0, \mathrm{t} 1, \mathrm{t} 2$, and $\mathrm{t} 3$ for the four diagnostic groups. $n=151$ due to missing values

IBI-subscale scores with diagnosis as between-subjects factor was conducted. This analysis showed an overall main effect of diagnosis $(F(15,534)=2.6, p=0.001)$. This indicates that patients from the diagnostic groups differed in their scores on the IBI-subscales at baseline (Table 2). Furthermore, this analysis yielded significant univariate main effects of diagnosis for IBI-Worrying $(F(3,180)=7.3, p<0.001)$, IBI-Rigidity $(F(3,180)=4.0, p=0.009)$, IBI-Need for Approval $(F(3,180)=3.8$, $p=0.01)$ and IBI-Problem Avoidance $(F(3,180)=3.8, p=0.01)$.

The double diagnosis group had the highest scores on the IBI-worrying subscale. The post-hoc analyses revealed that the mean scores in this subgroup were statistically significantly higher than those of the adjustment disorder group $(p \leq 0.0001)$ and the depression group $(p=0.02)$ but not significantly higher than the anxiety group $(p=0.56)$. The difference in means of the anxiety disorder group compared to other diagnostic groups was not statistically significant. The mean score of the depression group did also not differ significantly from the other diagnostic subgroups apart from the double diagnosis group.

The double diagnosis group had the highest scores on the IBI-rigidity subscale. Post-hoc analyses revealed that the difference of this diagnostic group with all other diagnostic groups was statistically significant ( $p$-values ranging from 0.01 to 0.02 ). Among these other diagnostic groups no statistically significant differences were found.

The double diagnosis group also had the highest scores on the IBI-Need for Approval, IBI-Problem Avoidance and on the IBI-Emotional irresponsibility subscale. The post-hoc analyses revealed that, for these subscales, the mean scores in this group did not statistically differ from any other diagnostic group. Nor were statistically significant differences found among those other diagnostic groups.

Stability over time of type of irrational beliefs was tested using MANOVAs, with repeated measurements of IBI-subscale scores with diagnosis as between-subjects 
factor and time as the within-subject factor. The MANOVA revealed statistically significant main effects for time in the following subscales: IBI-Worrying $(F(3,447)=12.1, \quad p<0.0001), \quad$ IBI-Need for Approval $(F(3,444)=7.1$, $p<0.0001)$, IBI-Problem Avoidance $(F(3,444)=4.0, p=0.008)$ and IBI-Emotional Irresponsibility $(F(3,441)=3.2, p=0.02)$. As can also be seen in Table 2 , this main effect over time is due to a decrease over time in the scores on these subscales. For the subscale IBI-Rigidity such a significant main effect was not found $(F(3,444)=0.7, p=0.57)$, the scores on this subscale appear stable over time (Table 2).

A statistically significant interaction effect of diagnosis and time was found for IBI-Emotional irresponsibility. Tukey post-hoc analysis showed that this effect was due to a statistically significant difference between the adjustment disorder group and the double diagnosis group ( $p=0.03$ ). As can be seen in Table 2, the mean scores in the double diagnosis group first show an increase before they decrease compared to baseline level. For all other subscales, no statistically significant interaction effect of diagnosis and time was found. This suggests that the decrease over time for these types of irrational beliefs is the same for the four diagnostic groups.

\section{Relation between Change in Irrational Beliefs and Symptom Recovery}

In order to describe the relation between change in irrationality and symptom recovery, employees were classified into three groups based upon their change in IBI total score between $\mathrm{t} 0$ and $\mathrm{t} 3$. Subsequently, overall differences of these groups on the three DASS-subscales at $\mathrm{t} 0, \mathrm{t} 1, \mathrm{t} 2$ and $\mathrm{t} 3$ were examined with three separate repeated measurements MANOVAs. IBI change group was used as betweensubjects factor and time as within-subject factor. The three IBI-change groups, based on the tertile scores, differed substantially in their mean change in IBI-total score, while no statistically significant differences were observed between IBI-total scores at baseline $(F(2,154)=2.7, p=0.07)$. The first group had a mean increase of 6.0 (IBI-increase group), the second group showed a mean decrease of 7.7 (IBI-small decrease group) and the third group was found to have a mean decrease of 22.2 (IBI-substantial decrease group). A time by IBI-change group interaction effect was statistically significant for depressive $(F(6,294)=3.4, p=0.003)$, anxiety $(F(6,294)=2.4, p=0.03)$ and stress symptoms $(F(6,314)=3.7$, $p=0.001)$. The IBI-increase group showed the smallest reduction in depression, anxiety and stress symptom levels followed by the IBI-small decrease group (Fig. 2). The IBI-substantial decrease group had the largest reduction in symptom levels over time. This means that a change in irrational beliefs is related to the magnitude of symptom recovery.

\section{Discussion}

This study showed that employees with both a depression and an anxiety disorder have the highest level of irrational beliefs, followed by employees with only anxiety 



Fig. 2 Mean scores of DASS subscales at $\mathrm{t} 0, \mathrm{t} 1, \mathrm{t} 2$, and $\mathrm{t} 3$ for three IBI-change groups. $n=151$ due to missing values

disorders. This effect remains over the course of one year. Employees with a depression had lower levels of irrationality than employees with anxiety disorders, but higher levels than the group with adjustment disorders. This is in line with the 
expectation that patients with more severe diagnoses within the group of common mental disorders endorse higher levels of irrational beliefs. The finding of higher levels of irrationality in patients with anxiety disorders compared to depressive symptoms was somewhat surprising. Chang and Bridewell (1998) found that higher levels of irrational beliefs were more related to depressive then to anxiety symptoms in their student population.

In contrast to our expectations, we did not find any type of irrational belief as being most pronounced in certain disorders. The double diagnosis group had higher scores than the other diagnostic groups on all subscales. The finding of Taghavi and colleagues (2006) and Koopmans and colleagues (1994) that emotional irresponsibility is specific for depression was not corroborated. Nor was the specificity of need for approval in anxiety (Koopmans et al. 1994; Ciarriochi 2004). However, future studies should reveal whether the IBI questionnaire is inappropriate for measuring disorder-specific beliefs, or whether the diagnostic groups differ only in level of irrationality.

The longitudinal design of this study provided the means for an examination of the course of irrationality. As in earlier studies on depression alone, change in irrational cognitions was associated with change in symptoms (Oei and Free 1995). Although it is not a direct test of the REBT theory of change it does substantiate the assumption that symptom change is mediated by a change in irrationality.

We found that participants with an increase in IBI-scores over 12 months had the smallest reduction in symptoms and that participants who had a decrease in their level of irrational beliefs over 12 months had the largest reduction. However, if irrational beliefs are responsible for a decrease in symptoms, one would have expected that in a group with participants experiencing an increase in irrational beliefs, the symptom levels would have increased as well. One possible explanation for this unexpected finding is that such an increase of symptoms occurred at a time point later than our last measurement. As for now the question whether a decrease in irrationality precedes symptom recovery remains unanswered. It has been argued that irrational cognitions and symptoms are both influenced by an unknown common cause (Burns and Spangler 2001). In future research, structural equation modelling might be used to thoroughly investigate the temporal relationship between beliefs and symptoms.

Our group of patients with adjustment disorders had a level of irrational beliefs (mean IBI-total score 140.6) comparable to those found in a community sample (mean score 140.9) in a previous study (Koopmans et al. 1994). The level of irrationality in the other diagnostic groups was higher. This is in agreement with the notion that anxiety disorders and depression are considered more severe disorders than adjustment disorders (Casey et al. 2001). At follow-up (T3), patients from the adjustment disorder group had even lower levels of irrationality (mean score 132.4) than normal controls. Participants with an anxiety disorder or a double diagnosis had irrationality scores higher than normal controls at follow-up (144.2 and 154.1, respectively). This could mean that they had not yet recovered sufficiently. Another explanation is that these patients had a stable tendency to have irrational beliefs, which made them prone to relapse. 
In conclusion, groups with different diagnoses differed more in level of irrationality than in type of irrational beliefs and this effect was found at baseline and over the course of one year. Depression and anxiety disorders showed higher levels of irrationality than adjustment disorders. Furthermore, the whole group showed a decrease in irrational beliefs over time, while no differential effects for diagnosis were found. In accordance with expectations, the magnitude and direction of change in irrationality was associated with the magnitude of symptom recovery. These findings suggest that CBT may encompass similar cognitive interventions for all common mental disorders. Furthermore, cognitive interventions seem to be most appropriate for patients with depression and anxiety disorders. To a certain extent, the association between changes in irrational beliefs and symptoms supports the use of cognitive interventions in CBT. A challenging task for future research will be to answer the question of whether a decrease in irrational beliefs is a prerequisite for symptom recovery.

Acknowledgements Grants were obtained from: The Netherlands Organization for Scientific Research (NWO): Netherlands Concerted Research Action "Fatigue at Work"; and the Foundation for Replacement and Occupational Health in Education (Stichting Vf/BGZ).

Open Access This article is distributed under the terms of the Creative Commons Attribution Noncommercial License which permits any noncommercial use, distribution, and reproduction in any medium, provided the original author(s) and source are credited.

\section{References}

Antony, M. M., Bieling, P. J., Cox, B. J., Enns, M. W., \& Swinson, R. P. (1998). Psychometric properties of the 42-item and 21-item versions of the Depression Anxiety Stress Scales in clinical groups and a community sample. Psychological Assessment, 10, 176-181.

American Psychiatric Association (1994). Diagnostic and statistical manual of mental disorders, fourth edition (DSM-IV). Washington: APA.

Beck, A. T. (1976). Cognitive therapy and the emotional disorders. New York: International Universities Press.

Brown, T. A., Chorpita, B. F., Korotitsch, W., \& Barlow, D. H. (1997). Psychometric properties of the Depression Anxiety Stress Scales (DASS) in clinical samples. Behaviour Research and Therapy, 35, 79-89.

Burns, D. D., \& Spangler, D. L. (2001). Do changes in dysfunctional attitudes mediate changes in depression and anxiety in cognitive behavioral therapy? Behavior Therapy, 32, 337-369.

Casey, P., Dowrick, C., \& Wilkinson, G. (2001). Adjustment disorders: Fault line in the psychiatric glossary. The British Journal of Psychiatry, 179, 479-481.

Chang, E. C. (1997). Irrational beliefs and negative life stress: Testing a diathesis-stress model of depressive symptoms. Personality and Individual Differences, 22, 115-117.

Chang, E., \& Bridewell, W. (1998). Irrational beliefs, optimism, pessimism, and psychological distress: A preliminary examination of differential effects in a college population. Journal of Clinical Psychology, 54, 137-142.

Chang, E. C., \& D'Zurilla, T. J. (1996). Irrational beliefs as predictors of anxiety and depression in a college population. Personality and Individual Differences, 20, 215-219.

Ciarrochi, J. (2004). Relationships between dysfunctional beliefs and positive and negative indices of well-being: A critical evaluation of the Common Beliefs Survey-III. Journal of Rational-Emotive and Cognitive-Behavior Therapy, 22, 171-188.

Clara, I. P., Cox, B. J., \& Enns, M. W. (2001). Confirmatory factor analysis of the depression-anxietystress scales in depressed and anxious patients. Journal of Psychopathology and Behavioral Assessment, 23, 61-67. 
David D., Szentagotai, A., Eva, K., \& Macavei, B. (2005). A synopsis of Rational-Emotive Behavior Therapy (REBT); fundamental and applied research. Journal of Rational-Emotive and CognitiveBehavior Therapy, 23, 175-221.

Department of Health (2001). Treatment choice in psychological therapies and counselling. London: HMSO.

De Zwart, B. C. H., Broersen, J. P. J., Van der Beek, A. J., Frings-Dresen, M. H. W., \& van Dijk, F. J. H. (1997). Occupational classification according to work demands: An evaluation study. International Journal of Occupational Medicine and Environmental Health, 10, 283-295.

Ellis, A. (1977). The basic clinical therapy of rational emotive therapy. In A. Ellis \& R. Grieger (Eds.), Handbook of rational-emotive therapy. New York: Springer.

Ellis, A. (1995). Changing rational-emotive therapy (RET) to rational emotive behavior therapy (REBT). Journal of Rational-Emotive and Cognitive-Behavior Therapy, 13, 85-89.

Emanuels-Zuurveen, L., \& Emmelkamp, P. M. G. (1996). Individual behavioural-cognitive therapy vs. maital therapy for depression in maritally distressed couples. British Journal of Psychiatry, 169, $181-188$.

Emmelkamp, P. M., \& Beens, H. (1991). Cognitive therapy with obsessive-compulsive disorder: A comparative evaluation. Behavior Research and Therapy, 29, 293-300.

Emmelkamp, P. G., Mersch, P., Vissia, E., \& van der Helm, M. (1985). Social phobia: A comparative evaluation of cognitive and behavioural interventions. Behavior Research and Therapy, 23, 365369.

Engels, G. I., Garnefsky, N., \& Diekstra, F. W. (1993). Efficacy of rational-emotive therapy: A quantitative analysis. Journal of Consulting and Clinical Psychology, 61, 1083-1090.

Fenig, S., Levav, I., Kohn, R., \& Yelin, N. (1993). Telephone vs. face-to-face interviewing in a community psychiatric survey. American Journal of Public Health, 83, 896-898.

Kassinove, H., \& Eckhardt, C. I. (1994). Irrational beliefs and self-reported affect in Russia and America. Personality and Individual Differences, 16, 133-142.

Koopmans, P. C., Sanderman, R., Timmerman, I., \& Emmelkamp, P. M. G. (1994). The Irrational Beliefs Inventory (IBI): Development and psychometric evaluation. European Journal of Psychological Assessment, 10, 15-27.

Lipsky, M., Kassinove, H., \& Miller, N. (1980). Effects of rational-emotive therapy, rational role reversal and rational-emotive imagery on the emotional adjustment of community mental health center patients. Journal of Consulting and Clinical Psychology, 48, 366-374.

Lovibond, S. H., \& Lovibond, P. F. (1993). Manual for the Depression Anxiety Stress Scales (DASS). University of New South Wales.

Lovibond, P. F., \& Lovibond, S. H. (1995). The structure of negative emotional states: Comparison of the Depression Anxiety Stress Scales (DASS) with the Beck depression and anxiety inventories. Behaviour Research and Therapy, 33, 335-343.

Lyons, L. C., \& Woods, P. J. (1991). The efficacy of rational-emotive therapy: A quantitative review of the outcome research. Clinical Psychology Review, 11, 357-369.

Malouff, J. M. (1992). Examination of the relationship between irrational beliefs and state anxiety. Personality and Individual Differences, 13, 451-456.

Nieuwenhuijsen, K., De Boer, A. G., Verbeek, J. H., Blonk, R. W., \& van Dijk, F. J. (2003). The Depression Anxiety Stress Scales (DASS): Detecting anxiety disorder and depression in employees absent from work because of mental health problems. Occupational and Environmental Medicine, 60(Suppl 1), I77-I82.

Oei, T. P. S., \& Free, M. L. (1995). Do cognitive behaviour therapies validate cognitive models of mood disorders? A review of the empirical evidence. International Journal of Psychology, 30, 145-179.

Rohde, P., Lewinsohn, P. M., \& Seeley, J. R. (1997). Comparability of telephone and face-to-face interviews in assessing axis I and II disorders. American Journal of Psychiatry, 154, 1593-1598.

Simon, G. E., Revicki, D., \& VonKorff, M. (1993). Telephone assessment of depression severity. Journal of Psychiatric Research, 27, 247-252.

Taghavi, M. R., Goodarzi, M. A., Kazemi, H., \& Ghorbani, M. (2006). Irrational beliefs in major depression and generalized anxiety disorders in an Iranian sample: A preliminary study. Perceptual and Motor Skills, 102, 187-196.

World Health Organization (1997). Composite International Diagnostic Interview (CIDI), version 2.1. WHO. 